\begin{tabular}{|c|l|}
\hline Title & Detection of molecular charge dynamics through current noise in a GaA s based nanowire FET \\
\hline Author(s) & Inoue, Shinya; Kuroda, Ryota; Yin, Xiang; Sato, Masaki; Kasai, Seiya \\
\hline Citation & $\begin{array}{l}\text { Japanese Journal of A pplied Physics(JJAP), 54(4S), O4DN07 } \\
\text { https://doi.org/L0.7567/JJAP.54.04DNO7 }\end{array}$ \\
\hline Issue Date & 2015-04 \\
\hline Doc URL & http://hdl.handle.net/2115/66631 \\
\hline Rights & ○ 2015 The Japan Society of A pplied Physics \\
\hline Type & article (author version) \\
\hline File Information & JJAP2015_54_SS14190.pdf \\
\hline
\end{tabular}

Instructions for use 


\title{
Detection of Molecular Charge Dynamics through Current Noise in A GaAs-based Nanowire FET
}

\author{
Shinya Inoue, Ryota Kuroda, Xiang Ying, Masaki Sato, and Seiya Kasai* \\ Research Center for Integrated Quantum Electronics, and Graduate School of Information \\ Science and Technology, Hokkaido University, Sapporo 060-8628, Japan \\ E-mail: kasai@ rciqe.hokudai.ac.jp
}

Detection of static and dynamic molecular charge state using a GaAs-based nanowire field-effect transistor (FET) was investigated. Tetraphenylporphyrin (TPP) was put on the device as target molecules. After coating TPP on the FET, the drain current obviously decreased. On the other hand, the current largely increased by 405-nm light irradiation, indicating that TPP worked as photo-excited donor. The light irradiation on the FET also induced Lorentzian noise component, which was superimposed onto conventional $1 / f$ noise. These behaviors were not seen in the gateless nanowire even with TPP. Obtained results indicated that electrical interaction between TPP and the nanowire was enhanced when a metal gate existed, although the channel was protected from TPP by the gate metal. We discuss the observed behaviors on the basis of a model where only TPP in the gate periphery modulated the channel potential and the drain current. 


\section{Introduction}

Detection of the charge state of a single molecule is important for chemistry, biology, medicine, health care, electronic, etc. Although scanning probe-based techniques have been already available as very powerful tools for such purposes, ${ }^{1-3)}$ there are still strong demands on more simple, compact, and easy-to-use way. Dynamic detection is also expected to provide further useful information of the target molecule, however, high-speed sensing of very weak signal is usually quite difficult. Recently, semiconductor nanowires and carbon nanotubes (CNTs) have been intensively investigated to detect the molecular charge states. These materials are very sensitive to surface charge because of high surface-to-bulk ratio. ${ }^{4-6)}$ Selective biochemical sensing has been successfully demonstrated using $\mathrm{CNTs}^{7-9)}$ and Si nanowires ${ }^{10-12)}$. In this paper, we investigate a GaAs-based nanowire field-effect transistor (FET) for detection of the static and dynamic charge state of the molecule. The device used in this study consists of AlGaAs/GaAs heterostructure ${ }^{13-16)}$ which has good potential for highly sensitive detection of weak signal. High quality AlGaAs/GaAs heterointerface provides high electron mobility and low noise capability. ${ }^{17,18)}$ The drain current can be adjusted by the gate suitable for detecting the molecular charge state. For detecting the molecular charge dynamic, we focus on the drain current noise of the FET. The GaAs-based nanowire FET is preferable to this approach, since the background noise in the device is much smaller than that in Si MOSFETs. ${ }^{19,20)}$

\section{Concept and setup}

Basic setup is schematically shown in Fig. 1(a). A GaAs-based nanowire FET was used as a charge detector. Target molecules were put on the device and a part of them directly contacted with the nanowire surface. In this study, tetraphenylporphyrin (TPP) was used as the molecular material. Porphyrin is known as an electroluminescent layer of the organic electroluminescent diode ${ }^{21,22)}$ and photo-excited donor in the dye-sensitized solar cell. $^{23,24)}$ Light absorption band of TPP is around $419 \mathrm{~nm}^{25)}$ Energy level diagram of PtPd, TPP, AlGaAs, and GaAs is shown in Fig. 1(b). PtPt was used for the gate metal of our device. The diagram suggests that photo-excited electron in TPP will be transferred to GaAs and TPP is expected to work as photo-excited donor for GaAs-based nanowire. A light emitting diode (LED) with the wavelength of $405 \mathrm{~nm}$ was place above the FET for 
exciting carriers in TPP. The tail of the spectrum peak overlapped the TPP absorption band. DC drain current, $I_{\mathrm{DS}}$, was measured as output of the detector FET. When the charge state of TPP is changed, $I_{\mathrm{DS}}$ is simultaneously changed. Charge interaction between the molecule and the device will take place stochastically and it is expected to appear as drain current noise. Generally $1 / f$ noise is dominant in the Schottky-gate GaAs-based nanowire FET, ${ }^{19}$ ) similar to the conventional FETs. However, when discrete charging and discharging occurs stochastically through discrete states in a molecule, it causes random telegraph signal (RTS) noise. ${ }^{26-28)}$ The RTS noise has a Lorentzian spectrum with $1 / f^{2}$ dependence, which is superimposed onto $1 / f$ noise.

Scanning electron microscope (SEM) image of the fabricated GaAs-based nanowire FET together with its layer structure is shown in Fig. 1(c). The device was fabricated on the $\mathrm{AlGaAs} / \mathrm{GaAs}$ heterostructure having two-dimensional electron gas (2DEG). Si delta doping was used to generate the 2DEG. The nanowire channel was formed by electron beam (EB) lithography and wet chemical etching. The nanowire length and width of the fabricated device were $4 \mu \mathrm{m}$ and $400 \mathrm{~nm}$, respectively. The Schottky gate length was $400 \mathrm{~nm}$. Electron mobility $\mu$ and sheet carrier density $n_{\mathrm{S}}$ of the 2DEG were $2700 \mathrm{~cm}^{2} / \mathrm{Vs}$ and $7 \times 10^{11} \mathrm{~cm}^{-2}$, respectively. For comparison, a gateless nanowire device having similar dimensions to the channel of the FET was also fabricated on the same chip. 20-nm thick TPP layer was put on the device by spin coating xylene-diluted TPP. From the size of TPP, the sheet density of the monolayer TPP directly contacting with the nanowire surface was estimated to be $1.2 \times 10^{14} \mathrm{~cm}^{-2}$. The dynamic change of $I_{\mathrm{DS}}$ was measured using a spectrum analyzer after amplifying the drain current by a low-noise amplifier (LNA) with a bandwidth of $500 \mathrm{kHz}$. Noise floor of the measurement system was sufficiently smaller than noise generated in the FET itself. All measurements were carried out at room temperature.

\section{Experimental results}

\subsection{DC drain current characteristics}

Measured drain current - drain voltage $\left(I_{\mathrm{DS}}-V_{\mathrm{DS}}\right)$ characteristics of a gateless nanowire and a nanowire FET are shown in Figs. 2(a) and 2(b), respectively. By coating TPP, the drain current decreased in both devices. This suggested that a part of electrons in 
the nanowire were transferred to TPP and the charge state of TPP reflected on the drain current. Slope of the $I_{\mathrm{DS}}-V_{\mathrm{DS}}$ curve in the low drain voltage region became gentle in both devices, which showed the increase of the channel access resistance owing to enhanced channel depletion by negatively charged TPP. In the saturation region, $I_{\mathrm{DS}}$ in the FET greatly decreased as compared with the gateless nanowire. Amount of the decrease of $I_{\mathrm{DS}}$ in the FET was $4 \mu \mathrm{A}$ at $V_{\mathrm{DS}}=1.2 \mathrm{~V}$, which was twice of that in the gateless nanowire. Decrease of the saturation current suggested the shift of the threshold voltage $V_{\text {th }}$ to the positive gate voltage side. This behavior contradicted the configuration of the devices; observed $V_{\text {th }}$ shift corresponded to decrease of electron density underneath the gate, however, TPP on the gate metal could not affect the channel covered with the metal.

Next we characterized the photoresponse of the FETs by LED light irradiation. Figure 3 shows measured transfer characteristics under light irradiation in the FET before and after TPP coating. Change of the drain current by light irradiation was not obvious in the FET without TPP as shown in Fig. 3(a), however the current was largely increased in the FET with TPP as shown in Fig. 3(b). By $1 \mathrm{~mW}$ light irradiation, the saturated drain current in the FET with TPP was found to recover to the value before TPP coating (not shown in Fig. 3(b)). We also found that the current in the gateless nanowire with TPP recovered similarly by light irradiation. The observed current change showed that the electron in TPP was transferred to the GaAs nanowire, namely TPP worked as photo-excited donor. In the case of the Si nanowire, a similar result was obtained by Winkelmann et al., ${ }^{29)}$ meanwhile Choi et al. showed that the current was decreased by light irradiation. ${ }^{30)}$ The doping type might be sensitive to the work function of TPP and detailed energy level alignment with the semiconductor. As the LED power increased, the transfer characteristics of the FET with TPP changed in two ways: increase of the saturated drain current and $V_{\text {th }}$ shift to negative gate voltage side. The former was explained by the decrease of the channel access resistance owing to electron supply to the channel from photo-excited TPP. The observed $V_{\text {th }}$ shift suggested that the carrier density below the gate was increased by light irradiation. When the LED power was increased to $1 \mathrm{~mW}, V_{\text {th }}$ decreased less than that in the FET without TPP. This confirmed that excess electrons were supplied from TPP. However, the observed behavior also contradicted that expected from the configuration of the device. The charge in TPP should be screened by the gate metal 
and they would not affect the carriers underneath the gate. Photo-excitation of electrons underneath the metal gate was rejected, since the increase of the drain current in the gateless nanowire without TPP was small as shown in Fig. 3(a).

\subsection{Drain current noise}

Measured low-frequency drain current noise spectra under light irradiation are summarized in Fig. 4. The gateless nanowire without TPP showed a typical $1 / f$ noise as shown in Fig. 4(a). Any significant effect of the light irradiation was not seen. After coating TPP, noise power was slightly increased, whereas the $1 / f$ spectrum was maintained as shown in Fig. 4(b). Again the light did not give any significant effect on the noise even with TPP. The nanowire FET without TPP also showed $1 / f$ noise as shown in Fig. 4(c). The noise power was one order of magnitudes larger than the gateless nanowire. The noise in the fabricated nanowire FET was always larger than that in the gateless nanowire. The light irradiation on the FET without TPP did not significantly change the noise spectra. In case of $1 \mathrm{~mW}$ light, the spectrum was partly increased around $10 \mathrm{~Hz}$. It might be caused by generation-recombination through deep traps in the AlGaAs, however the origin is not clear yet. On the other hand, with TPP, the noise spectrum in the FET was clearly changed by light irradiation as shown in Fig. 4(d). The noise power increased as the light power increased. A kink appeared and the slope in the high frequencies became steeper than $1 / f$. The kink became obvious as the light power increased and its position shifted to higher frequency simultaneously.

Noise spectra from the FET with TPP could be decomposed to $1 / f$ and Lorentzian components as shown in the inset of Fig. 5. The existence of a Lorentzian component indicates that RTS noise appeared and the random discrete charging and discharging event took place in the system. Figure 5 summarizes the corner frequency and the power of Lorentzian component obtained by fitting each noise spectrum in Fig. 4(d). The corner frequency was monotonically increased from $110 \mathrm{~Hz}$ to $1,700 \mathrm{~Hz}$ with the increase of the LED light power, corresponding time constant of $1.4 \mathrm{~ms}$ and $90 \mu \mathrm{s}$, respectively.

\section{Discussion}


The effect of TPP on DC I- $V$ characteristics was remarkable when the metal gate existed on the nanowire. Furthermore, the threshold voltage shift arose from TPP, although the metal gate protected the channel from TPP. We confirmed that TPP was uniformly coated on the device by using optical microscope and atomic force microscope (AFM). To explain the complicated behaviors, we considered a model in which TPP only in the gate periphery modulated the channel potential and controlled the drain current as shown in Fig. 6(a). In the dark condition, TPP in the gate periphery depleted the channel locally and formed high resistance regions that limited the drain current. This local effect of TPP took place because the AlGaAs surface potential was changed by the large work function of the PtPd gate metal through TPP. Regarding TPP as a very thin insulator, TPP that contacted with both the metal and the nanowire was considered to form a tunnel metal-insulator-semiconductor (MIS) structure as shown in Fig. 6(b). It is known that the correlation between the metal work function and the surface potential of the semiconductor in the tunnel MIS structure is usually higher than that in the direct metal-semiconductor (MS) contact, since high-density interface states are generated in the MS interface and the semiconductor surface Fermi level is almost fixed at the charge neutrality level. ${ }^{31)}$

From the transfer characteristics in Fig. 3(a), the series resistance in the FET without TPP in the dark condition was evaluated to be $38 \mathrm{k} \Omega$, which well agreed with the resistance of $36 \mathrm{k} \Omega$ estimated from the carrier density, mobility, and structure dimensions. The series resistance was estimated to be $100 \mathrm{k} \Omega$ in the FET with TPP in the dark. Assuming that the increase of the resistance of $64 \mathrm{k} \Omega$ was caused by the localized TPP in the gate periphery, the local carrier density estimated to be $10^{8} \mathrm{~cm}^{-2}$. This density was much smaller than $n_{\mathrm{S}}$ of $2 \mathrm{DEG}$ of $7 \times 10^{11} \mathrm{~cm}^{-2}$, suggesting that the electrons below the gate periphery were completely depleted. However only a few negatively charged TPP was necessary to achieve such local depletion. This value was not so far from the number of TPP existing in the gate periphery of 400, estimated from the sheet density of TPP of $1.2 \mathrm{x}$ $10^{14} \mathrm{~cm}^{-2}$ and the area of the gate edge given by size of TPP $\mathrm{x}$ the nanowire width $W$. The transfer characteristics in Fig. 3(b) also showed the $V_{\text {th }}$ shift simultaneously. In general, $V_{\text {th }}$ in the FET is controlled by the carrier density in the gate edge on the source side. ${ }^{31)}$ The drain current in saturation is dominated by carrier injection from the source into the channel below the gate. Although TPP located in the gate periphery, its electrostatic effect 
persisted to the channel in the gate edge. To cancel the depletion in the gate periphery, positive excess gate voltage was necessary and then $V_{\text {th }}$ was increased in the FET with TPP.

The potential configuration shown in Fig. 6(b) indicated that the increase of the drain current by the light irradiation in the FET with TPP was caused by the decrease of the depletion in the gate periphery. The surface depletion region was a potential valley for the holes. Photo-excited holes were accumulated in the valley, on the other hand the excited electrons were emitted to the channel as shown in Fig. 6(c). The localized holes did not carry the drain current, but bended the surface potential of the nanowire down, which decreased the potential barrier for the electrons. This reduced the high resistance in the gate periphery. Thus the drain current was recovered by light irradiation.

The band diagrams in Figs. 6(b) and 6(c) also suggested that the observed photo-excited Lorentzian noise in Fig. 4(d) arose from the sequence of the generation of an electron-hole pair in TPP and its relaxation. A key point was that the time constants of the hole relaxation was longer than that of the electron emission. The potential configurations in the gate periphery shown in Fig. 6(c) indicated that the electron emission occurred promptly. However, even after the hole was emitted from TPP and the charge state of TPP was relaxed, the hole still remained in the AlGaAs surface and the downward band bending was held. After that the hole was disappeared by the surface recombination through the surface states of the AlGaAs. Such multi-step process of the hole relaxation was expected to result in the longer time constant. These electron emission and hole relaxation were stochastic discrete charge events, thus their dynamic sequence should appear as RTS in the drain current.

Here we also emphasize that the dynamic charge state of TPP is well reflected on the drain current when TPP contacts with both the metal gate and the nanowire surface. The electrostatic potential of TPP on the metal contacting portion is fixed at the potential of the metal, corresponding to Dirichlet boundary condition. The potential on the nanowire-contacting side changes, when the charging or discharging of TPP takes place. Analyzing the equivalent circuit in Fig. 7(a), it is found that the channel potential in the FET, $V_{\mathrm{C}}$, depends on the charge of TPP $Q_{\mathrm{TPP}}$ as follows, 


$$
V_{C}=\frac{\left(C_{M}+C_{A}\right) Q_{C}+C_{A} Q_{T P P}}{C_{M} C_{A}+C_{A} C_{B}+C_{B} C_{M}}
$$

where $Q_{\mathrm{C}}$ is the charge of the channel carriers, $C_{\mathrm{M}}, C_{\mathrm{A}}$, and $C_{\mathrm{B}}$ are metal-TPP capacitance, TPP-channel capacitance, and FET body capacitance, respectively. On the other hand, when TPP does not contact with the metal as shown in Fig. 7(b), corresponding to Neumann boundary condition, we find that $V_{\mathrm{C}}$ is given by the next formula.

$$
V_{C}=Q_{C} / C_{B}
$$

This formula shows that $V_{\mathrm{C}}$ does not depend on $Q_{\text {TPP. }}$ Eqs. (1) and (2) explain that the charge state of TPP clearly reflects on the drain current in the FET rather than the gateless nanowire.

The analysis above clarifies that again only TPP contacting to the metal gate modulates the drain current dynamically. Therefore the FET could detect charge state of the limited number of TPP, even though a lot of TPP existed on the device. Then the number of TPP contributing the Lorentzian noise, $N$, is estimated from the noise intensity. The RTS noise power $S_{\mathrm{I}}(f)$ is expressed as follows, ${ }^{32,33)}$

$$
S_{I}(f)=\frac{\left(g_{m} N e / C_{G}\right)^{2}}{f_{C}\left(1+\left(2 \pi f / f_{C}\right)^{2}\right)}
$$

where $f_{\mathrm{C}}$ is corner frequency of the Lorentzian spectrum, $g_{\mathrm{m}}$ is transconductance, and $C_{\mathrm{G}}$ is the gate capacitance. Here we assume that the average time constants for the RTS high and low levels are the same, and the $C_{\mathrm{G}}$ is taken into account as a peripheral capacitance that exists in parallel to $C_{\mathrm{B}}$ in Eq. (1). In case of $1 \mathrm{~mW}$ light power in Fig. 5, for example, $N=$ 0.19 is obtained. This value indicates that a small number of TPP contribute to the observed Lorentzian noise. The small $N$ less than unity might be due to overestimate of the transconductance for the TPP charge event. The photon flux density at LED power of 1 $\mathrm{mW}$ corresponds to the rate of a photo-excitation of electron-hole pair of $20 \mathrm{~s}^{-1}$ per TPP. It is noted that the thickness of TPP layer was $20 \mathrm{~nm}$ and the rate for TPP contributing the 
drain current RTS noise must be much smaller than the evaluated value. Increasing the light power, the photo-excitation event occurred frequently, which would increase $f_{\mathrm{C}}$. Flattening the band banding of the nanowire surface seemed to also affect $f_{\mathrm{C}}$. The light power dependence of the Lorentzian noise power arose from either the increase of TPP contributing to the RTS noise or the decrease of the series resistance as shown in Fig. 3(b).

From the discussion above, It is expected that the charge state of further limited number of TPP can be detected by narrowing the nanowire. Use of small size nanowire also can provide high sensitivity owing to increased transconductance.

\section{Conclusions}

For detecting the molecular charge state, we characterized the static drain current and low-frequency drain current noise in the GaAs-based nanowire FET with TPP on its channel. It was found that the nanowire FET could reflect the charge state of TPP. TPP was found to work as photo-excited donor on the GaAs-based nanowire. Stochastic discrete charging and discharging of TPP with time constant of $90 \mu$ s to $1.4 \mathrm{~ms}$ was detected through drain current noise with $405 \mathrm{~nm}$ light irradiation. These behaviors were not seen in the gateless nanowire with TPP. Obtained results were explained by the model in which the charge state of TPP only in the gate periphery modulated the channel potential. It was found that the GaAs nanowire FET having a metal gate has a good possibility for detecting static and dynamic charge state of single molecules with a simple and compact manner.

\section{Acknowledgments}

The authors sincerely thank Prof. S. W. Hwang of Korea University for valuable discussion. This work was partly supported by Grant-in-Aid for Scientific Research on Innovative Areas "Molecular Architectonics: Orchestration of Single Molecules for Novel Functions" (\#25110001). 


\section{References}

1) M. F. Crommie, C. P. Lutz, and D. M. Eigler, Science 262, 218 (1993).

2) D. J. Müller and Y. F. Dufrêne, Nature Nanotech. 3, 261 (2008).

3) L. Gross, Nature Chem. 3, 273 (2011).

4) A. K. Wanekaya, W. Chen, N. V. Myung, and A. Mulchandani, Electroanalysis 18, 533 (2006).

5) S. Pud, F. Gasparyan, M. Petrychuk, J. Li, A. Offenhäusser, and S. A. Vitusevich, J. Appl. Phys. 115, 233705 (2014).

6) B. He, T. J. Morrow, and C. D. Keating, Current Opinion in Chemical Biology 12, 522 (2008).

7) J. N. Wohlstadter, J. L. Wilbur, G. B. Sigal, H. A. Biebuyck, M. A. Billadeau, L. Dong, A. B. Fischer, S. R. Gudibande, S. H. Jameison, J. H. Kenten, J. Leginus, J. K. Leland, R. J. Massey, and S. J. Wohlstadter, Adv. Mater. 15, 1184 (2003).

8) K. Besteman, J.-O Lee, F. G. M. Wiertz, H. A. Heering, and C. Dekker, Nano Lett. 3, 727 (2003).

9) K. Maehashi and K. Matsumoto, Sensors 9, 5368 (2009).

10) M. Seol, S. Choi, C. Kim, D. Moon, and Y. Choi, Nano Lett. 6, 183 (2012).

11) F. Patolsky, G. Zheng, and C. M. Lieber, Nature Protoc. 1, 1711 (2006).

12) F. Patolsky, G. Zheng, and C. M. Lieber, Nanomedicine 1, 51 (2006).

13) S. Kasai, K. Jinushi, H. Tomozawa, and H. Hasegawa, Jpn. J. Appl. Phys. 36, 1678 (1997).

14) M. Yumoto, S. Kasai, and H. Hasegawa, Appl. Surf. Sci. 190, 242 (2002).

15) M. Yumoto, S. Kasai, and H. Hasegawa, Jpn. J. Appl. Phys. 41, 2671 (2002).

16) Y. Shiratori and S. Kasai, Jpn. J. Appl. Phys. 47, 3086 (2008).

17) T. Mimura, Jpn. J. Appl. Phys. 44, 8263 (2005).

18) P. Chavarkar and U. Mishra, in RF and Microwave Semiconductor Device Handbook, ed. M. Golio, (CRC Press, Florida, 2002) p. 126.

19) K. Miura, Y. Shiratori, and S. Kasai, Jpn. J. Appl. Phys. 50, 06GF18 (2011).

20) T. Muramatsu, K. Miura, Y. Shiratori, Z. Yatabe, and S. Kasai, Jpn. J. Appl. Phys. 51, 06FE18 (2012).

21) Y. Sakakibara, S. Okutsu, T. Enokida, and T. Tani, Jpn. J. Appl. Phys. 38, L1472 
(1999).

22) M. Jamghouri, E. Mohajerani, M. M. Amini, and E. Najafi, Appl. Phys. A 114, 445 (2014).

23) S. Mathew, A. Yella, P. Gao, R. Humphry-Baker, B. F. E. Curchod, N. Ashari-Astani, I. Tavernelli, U. Rothlisberger, Md. Khaja Nazeeruddin, and M. Grätzel, Nature Chem. 6, $242(2014)$.

24) S. Karthikeyan and J. Y. Lee, J. Phys. Chem. A 117, 10973 (2013).

25) M. Lovcinský, J. Borecký, P. Kubát, and P. Jezek, Gen. Physiol. Biophys. 18, 107 (1999).

26) L. K. J. Vandamme and F. N. Hooge, IEEE Trans. on Electron Devices 55, 3070 (2008).

27) A. L. McWhorter, in Semiconductor Surface Physics, ed. R. H. Kingston (University of Pennsylvania Press, Pennsylvania, 1957) p. 207.

28) M. J. Kirton and M. J. Uren, Adv. Phys. 38, 367 (1989).

29) C. B. Winkelmann, I. Ionica, X. Chevalier, G. Royal, C. Bucher, and V. Bouchiat, Nano Lett. 7, 1454 (2007).

30) S.-J. Choi, Y.-C. Lee, M.-L. Seol, J.-H. Ahn, S. Kim, D.-I. Moon, J.-W. Han, S. Mann, J.-W. Yang, and Y.-K. Choi, Adv. Mater. 23, 3979 (2011).

31) S. M. Sze, Physics of Semiconductor Devices (Wiley, New York, 2006) 3rd ed.

32) N. Clément, K. Nishiguchi, A. Fujiwara, and D. Vuillaume, Nature Comm. 1, 92 (2010).

33) L. Guo, E. Leobandung, and S. Y. Chou, Science 275, 649 (1997). 


\section{Figure Captions}

Fig. 1. (a) Experimental set up, (b) energy band diagram of TPP, AlGaAs and GaAs, and (c) SEM image of a GaAs-based nanowire FET with the layer structure.

Fig. 2. $I_{\mathrm{DS}}-V_{\mathrm{DS}}$ characteristics in (a) gateless nanowire and (b) nanowire FET.

Fig. 3. Measured $I_{\mathrm{DS}}-V_{\mathrm{G}}$ characteristics in nanowire FET under LED light irradiation: (a) without TPP and (b) with TPP.

Fig. 4. Measured noise spectra at $V_{\mathrm{DS}}=0.8 \mathrm{~V}, V_{\mathrm{G}}=0.5 \mathrm{~V}$ : (a) gateless nanowire without TPP, (b) gateless nanowire with TPP, (c) nanowire FET without TPP, and (d) nanowire FET with TPP.

Fig. 5. Noise power and corner frequency of Lorentzian noise components as a function of LED power in nanowire FET with TPP.

Fig. 6. (a) Schematic of the channel condition in nanowire FET with TPP in the dark and equivalent resistances, (b) band diagrams for each resistance part, and (c) band diagram under light irradiation.

Fig. 7. Schematics of TPP on nanowire and corresponding equivalent circuits: (a) TPP in the gate periphery and (b) TPP on nanowire. 

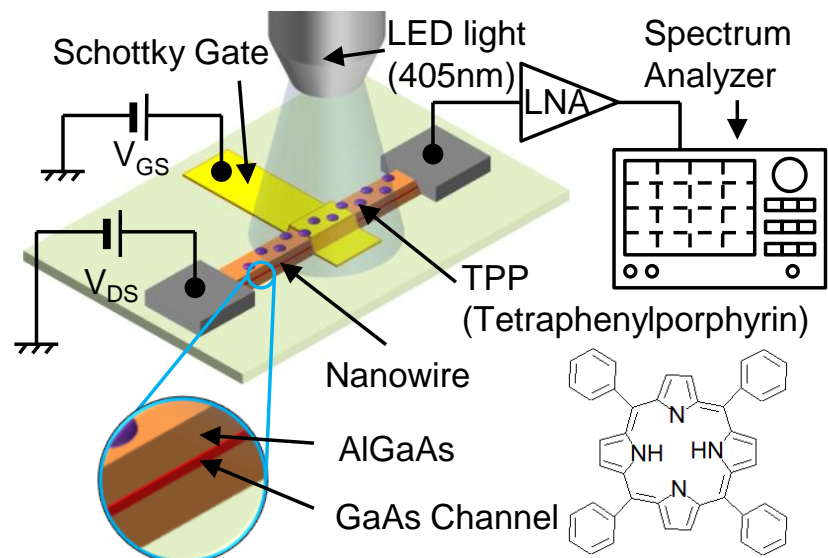

(a)
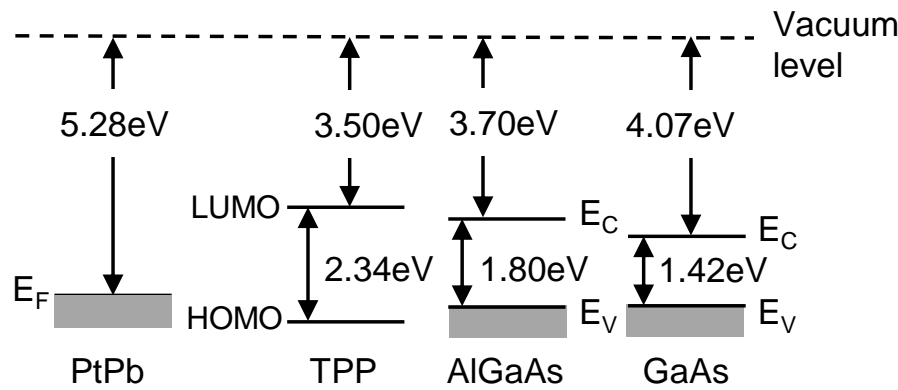

(b)
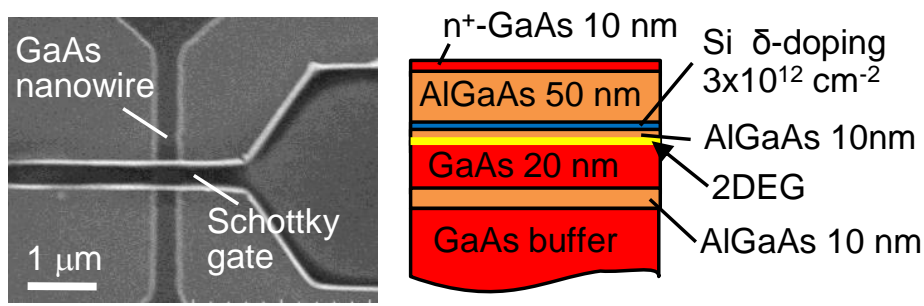

(c)

Fig. 1 Shinya Inoue et al. 

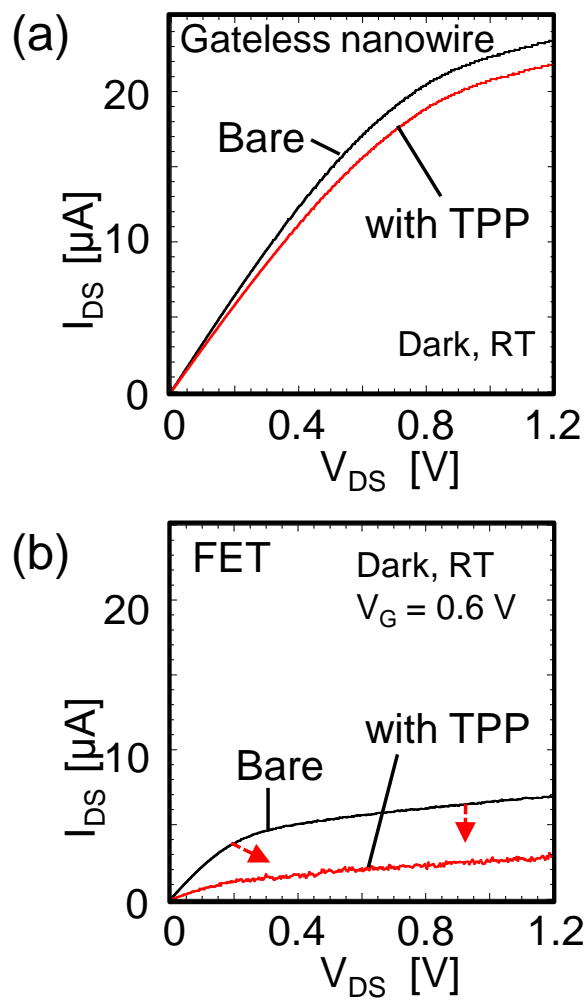

Fig. 2 Shinya Inoue et al. 
(a)

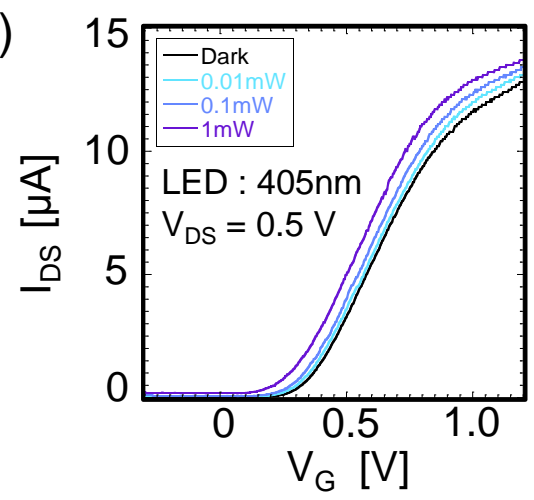

(b)

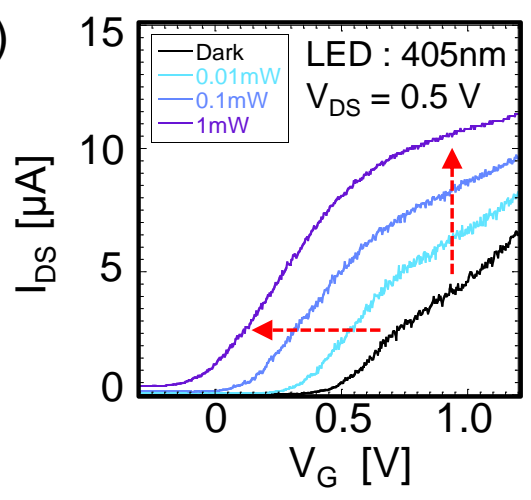

Fig. 3 Shinya Inoue et al. 
(a)

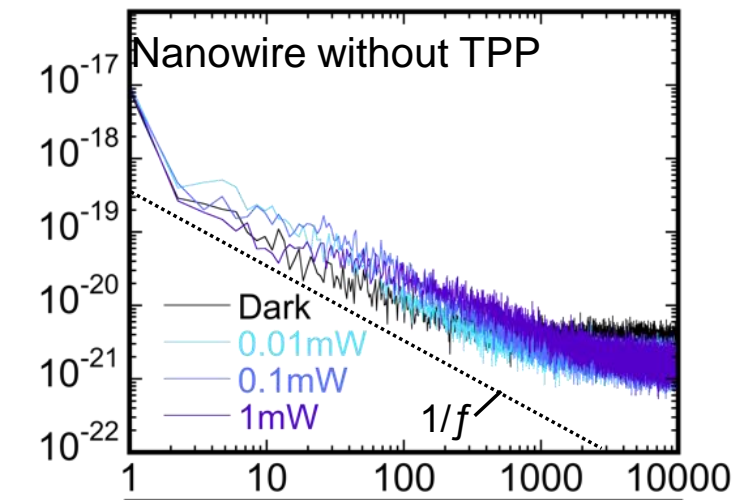

(b)

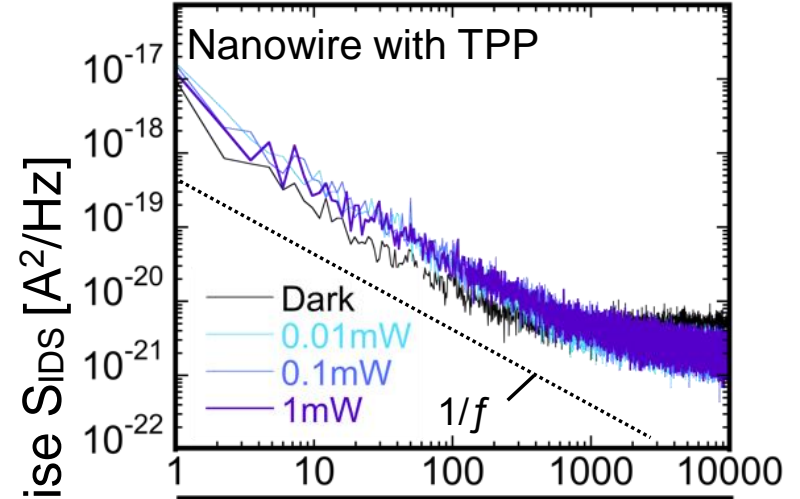

(c)

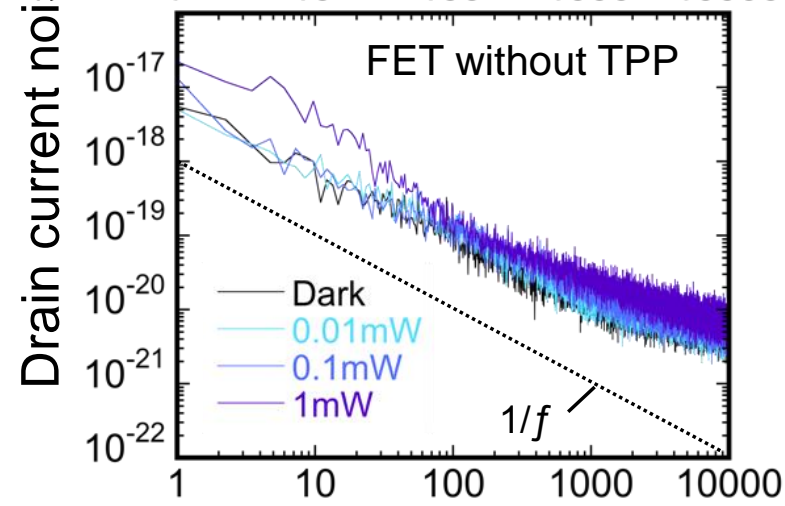

(d)

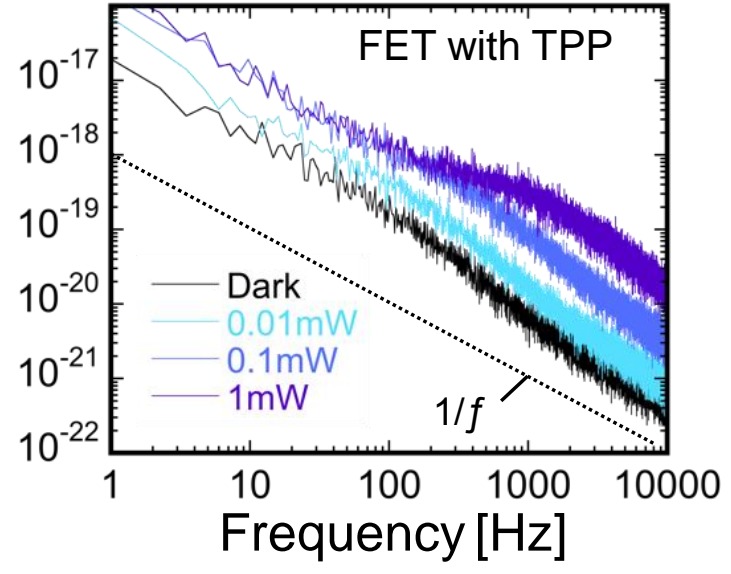

Fig. 4 Shinya Inoue et al. 


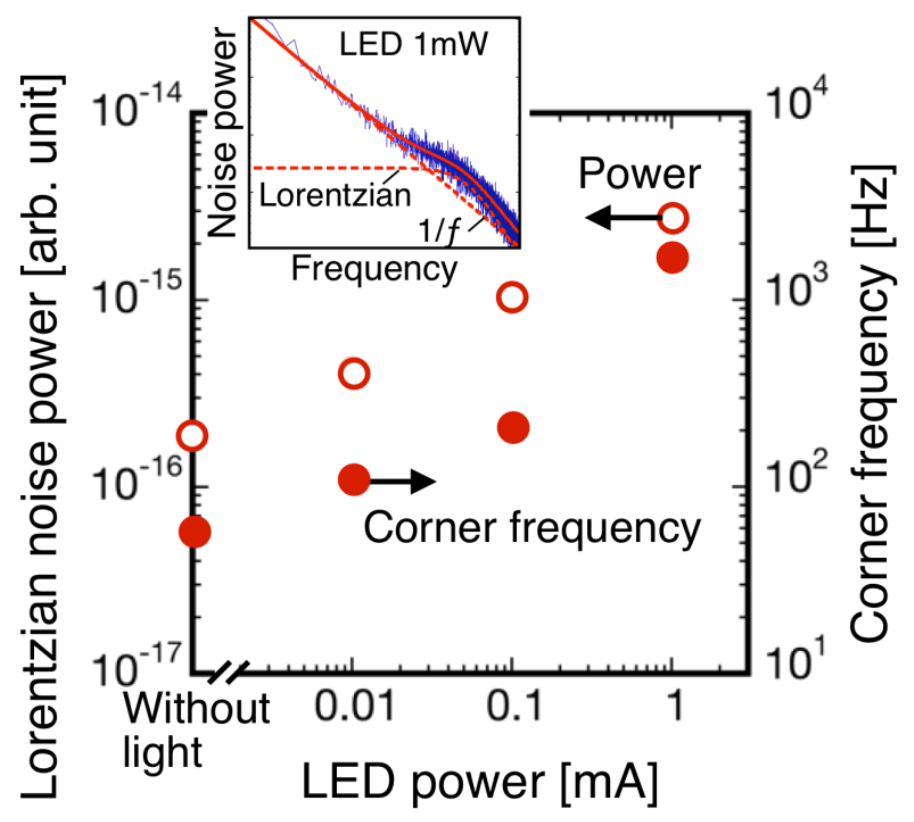

Fig. 5 Shinya Inoue et al. 
(a)

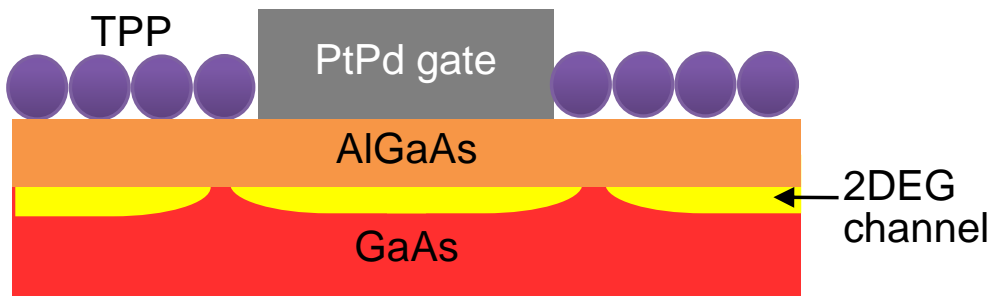

(b) Access region Gate periphery
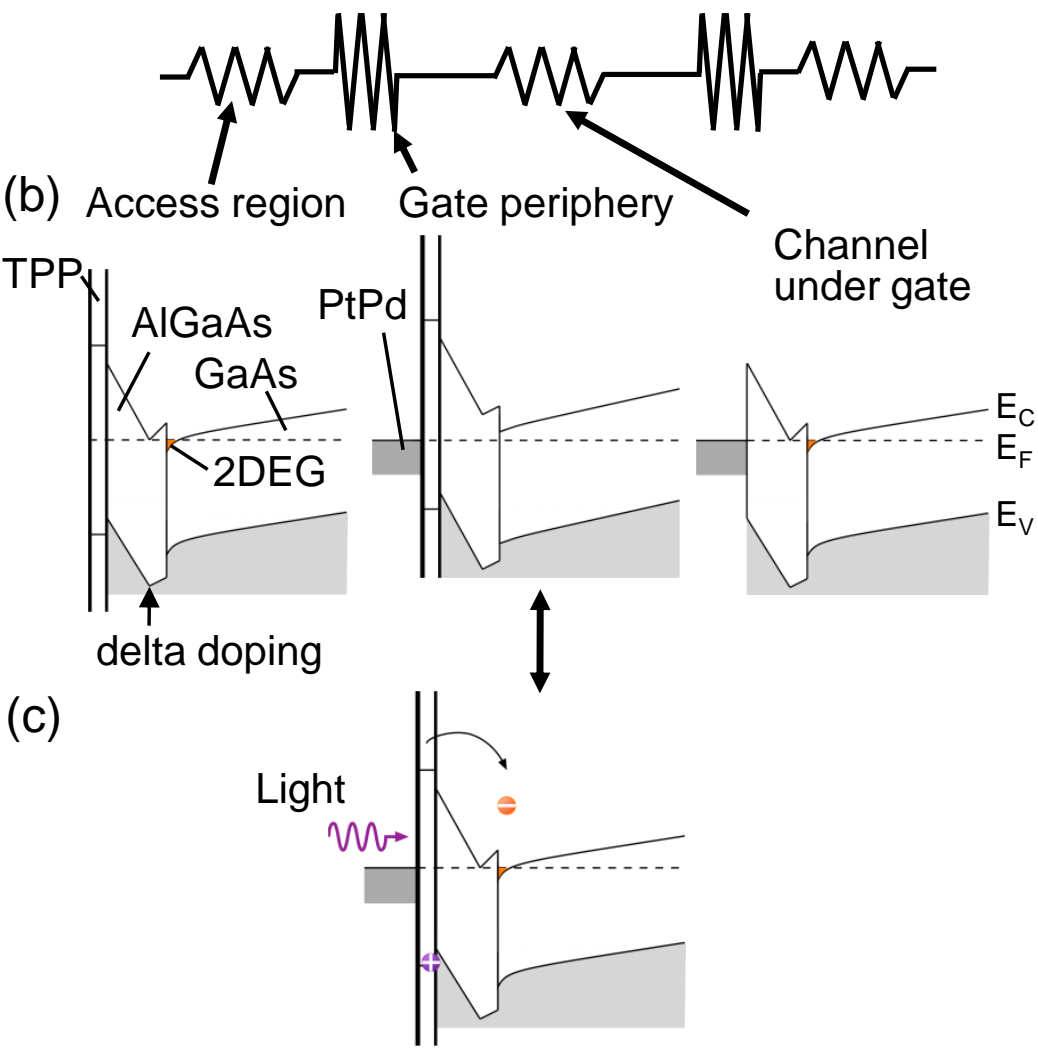

Fig. 6 Shinya Inoue et al. 
(a)

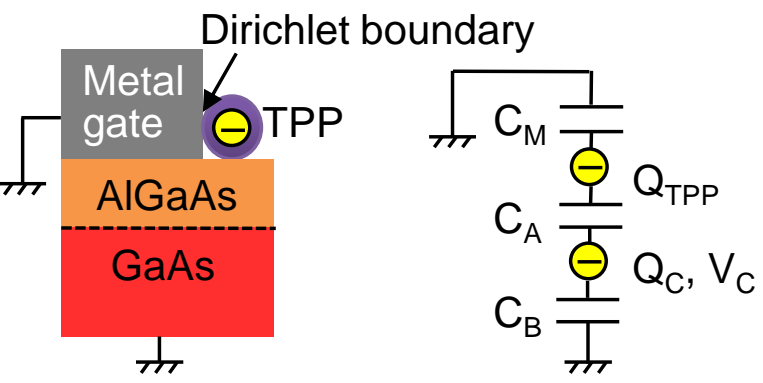

(b)

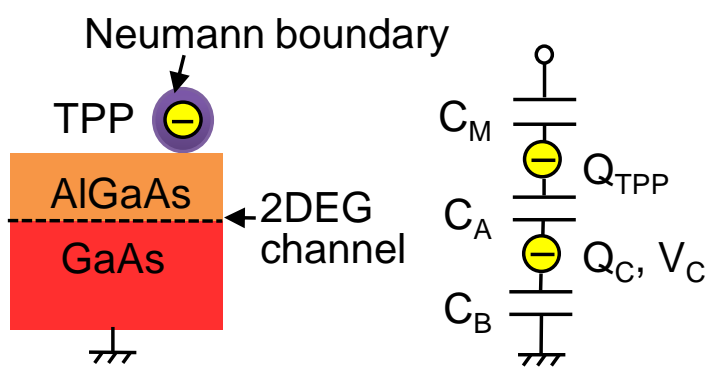

Fig. 7 Shinya Inoue et al. 